\title{
Isolation of chick retina cones and study of their diversity based on oil droplet colour and nucleus position
}

\author{
R. López-López • M. López-Gallardo • \\ M. J. Pérez-Álvarez • C. Prada
}

Received: 13 September 2007 / Accepted: 21 December 2007 / Published online: 12 February 2008

(C) The Author(s) 2008

\begin{abstract}
The chick retina has four morphological cone types that differ not only in shape, but also in the visual pigment in the outer segment, in the colour of the oil droplet in the inner segment and in synaptic connectivity. Neither the type of droplet nor the visual pigment has been definitively established for the four cone types. The main aim of the present work has been the isolation of entire live photoreceptors in order to study the oil droplet colour in each cone type and to quantify each type. We have improved an earlier retinal cell isolation method and obtained large numbers of entire cones. Principal cones ( $27 \%$ of the cones) possess a yellow or colourless droplet. Accessory cones ( $27 \%$ of the cones) all contain a small pale green droplet. Straight cones ( $44 \%$ of the cones) have a red, orange, yellow, or colourless droplet. Oblique cones $(1.66 \%$
\end{abstract}

This work was supported by grants from the Dirección General de Investigación, Ministerio de Educación y Ciencia of Spain (no. BFU2005-08786-C02-01) and from the Comunidad de Madrid, Spain (no. 920648/2006).

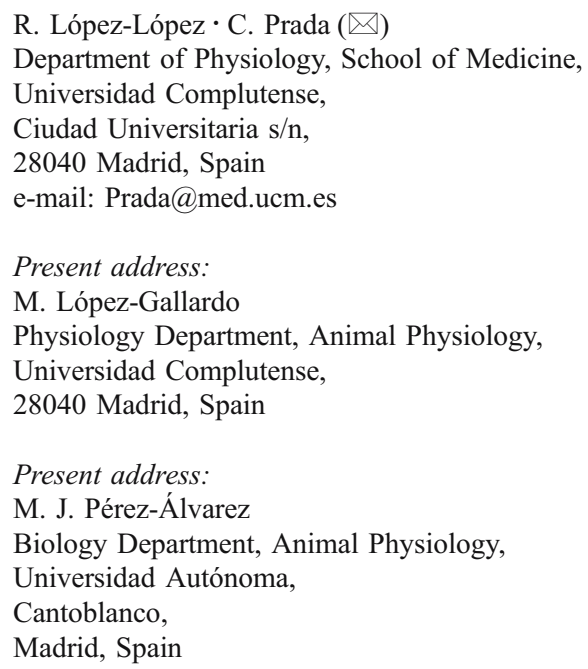

of the cones) all have a colourless droplet. We have found that straight cones with a red, orange, or yellow droplet differ in terms of the position of the nucleus and their percentage and conclude that they are distributed in three rows in the outer nuclear layer (ONL) of the central retina. Our study of 4,6-diamidino-2-phenylindole-stained retinal sections has revealed three rows of nuclei instead of the two currently thought to form the ONL. Together, our results show a larger cone diversity than previously known, suggest a larger functional diversity and provide an efficient method for isolating entire chick photoreceptors.

Keywords Retina - Dissociated cones · Oil droplet . Outer nuclear layer . Outer plexiform layer. Chick (White Leghorn)

\section{Introduction}

All vertebrate retinas, except those of the skate, have at least two morphological types of photoreceptors (rods and cones) that mediate dim-light vision, colour perception and fine-detail detection (Dowling 1987). Photoreceptor percentages vary with species, cones being a majority in the chick retina, viz. $86 \%$ cones versus $14 \%$ rods (Morris 1970). The Golgi studies by Ramón y Cajal (1892) in the chick retina revealed four morphological cone types: straight, diagonal (now termed oblique), principal and accessory. The straight cone has a relatively short, vertically oriented axon, whereas the diagonal cone has a long axon that bends steadily more to the side in its trajectory to become almost horizontally oriented in the inner stratum of the outer plexiform layer (OPL). The principal and accessory types form the "double cone", being connected to each other by gap junctions (Smith et al. 1985). The 
soma of the principal member is larger than that of the accessory member (Ramón y Cajal 1892), this member being easily identified in electron-microscopy preparations because its nucleus protrudes into the base of the inner segment across the limiting membrane (Morris 1970; Morris and Shorey 1967). Since the studies of Ramón y Cajal (1892), the oblique cone has been practically ignored. Indeed, with a few exceptions (Gallego 1976; Gallego et al. 1975; Gayoso et al. 1978; Mariani and Leure-duPree 1978), the studies of avian cones and particularly those of the chicken (Araki et al. 1984; Bowmaker et al. 1997; Bowmaker and Knowles 1977; Meyer and May 1973; Morris 1970; Morris and Shorey 1967; Oishi et al. 1990; Szel et al. 1985) refer to single cones and double cones, without distinguishing straight from oblique cones in the pool of single cones.

The soma of photoreceptors is almost completely occupied by the nucleus, the outer nuclear layer (ONL) being formed largely by photoreceptor nuclei, but also by outer processes of Müller cells. Current schemes show the ONL with two nuclei layers, with rods in the inner row and cones in both rows; the nuclei of the accessory and oblique cones are shown to be segregated to the outer row, whereas the nuclei of the principal and straight cones are restricted to the inner row (Gallego 1976; Morris and Shorey 1967). Studies with Drosophila mutants have shown the position of the photoreceptor nucleus to be developmentally controlled by mechanisms that also control photoreceptor fate and differentiation. Some molecules of these control mechanisms have been identified (Whited et al. 2004; Zhang et al. 2005). The nucleus position of chick photoreceptors might be controlled as in Drosophila photoreceptors.

The synaptic bodies of chick photoreceptors are located at three levels in the OPL (Gallego 1976; Gallego et al. 1975; Ramón y Cajal 1892). The outer (scleral) stratum contains the synaptic bodies of rods and the principal and accessory members of double cones. The intermediate stratum possesses the synaptic bodies of straight cones, whereas the inner stratum includes the synaptic bodies of the oblique cones. The synaptic bodies of cones form synapses with dendrites of horizontal cells at the three levels, whereas the synaptic bodies of rods form synapses with the axon terminal of horizontal cells (Gallego 1976; Gallego et al. 1975). Since each stratum of the OPL receives the dendrites of different types of bipolar cells (Ramón y Cajal 1892), double, straight and oblique cone types make contact with different bipolar cells, signifying that they participate in separate vertical pathways of information transmission in the retina. The stratification of the OPL of the chick retina also appears to be genetically controlled, since chicks with an autosomal recessive mutation that lies in chromosome 1 and that is responsible for the inherited ocular disease known as "retinopathy globe enlarged" (rge) show disorganization of the OPL and abnormal location of the endoplasmic reticulum of the photoreceptors as the first manifestation of the rge phenotype (Inglehearn et al. 2003; Montiani-Ferreira et al. 2005). Cone pedicles in this mutant are larger than those in the wild-type, irregular in shape and usually contain multivesicular bodies.

Chicken cones, as in most diurnal birds and some reptiles, have a coloured oil droplet in the outer end of the inner segment; this droplet acts as long-pass cut-off filter between the incident light and the visual pigment, determining the spectral content of light that reaches the visual pigment. Important functions such as the limitation of chromatic aberration and the enhancement of colour discrimination have been assigned to the coloured droplets (Bowmaker 1980; Hart 2001; Vorobyev 2003). Nevertheless, considerable disagreement exists among studies relating droplet colour to cone type (Bowmaker and Knowles 1977; Gallego 1976; Gayoso et al. 1978; Meyer and Cooper 1966; Meyer and May 1973; Morris and Shorey 1967; Oishi et al. 1990; Szel et al. 1985). This is partly attributable to the difficulty of identifying the cone types in fresh flat-mounted retinas, to date the only preparation in which the natural droplet colour can be viewed under light microscopy. However, several other factors are known to vary the observed droplet colour, such as the age of the chickens, their diet, the mode of microscopic observation (bright field, phase contrast or Nomarski interferential contrast) and the optical equipment of the microscope (type of lens and filters used), and are considered responsible for the discrepancies between these studies (López et al. 2005).

Since the droplet colour for all cone types identified by Ramón y Cajal (1892) in the chick retina has not been definitively established, the first objective of our work has been to isolate living entire chick photoreceptors to identify Ramón y Cajal's cone types and to study the colour of their droplets in fresh cell preparations. We have obtained dissociated entire cones of all types with their distinct droplet colours. Preliminary observations have shown variation in the position of the nucleus of straight cones with different droplet colours and, since the position of nucleus seems to be related to photoreceptor identity, our second objective has been to study straight cone diversity according to the nucleus position and oil droplet colour to determine their percentages. We reveal straight cones with the nucleus at three levels with respect to the outer limiting membrane (OLM; outer, middle and inner) differing by the droplet colour. Because we have found three levels of nucleus position, whereas chick photoreceptors have previously been considered to be arranged in two layers throughout the retina, our third objective has been to study 
the outer nuclear layer by using thin retinal sections $(5-8 \mu \mathrm{m})$ fixed with paraformaldehyde and mounted for nuclei staining with 4,6-diamidino-2-phenylindole (DAPI). We show that, in the central and equatorial retinal areas, photoreceptors are arranged in the outer, middle and inner rows, whereas in the most peripheral retina, they are arrayed in two rows, or even in one row. We have also established that the principal cones and straight cones with a red droplet lack an axon, contrary to the current idea that all these cones have an axon.

\section{Materials and methods}

\section{Animals}

Fertilized eggs from White Leghorn chickens (Gallus gallus domesticus) were obtained from a local supplier (Avícola Grau, Madrid) and incubated at $37.5^{\circ} \mathrm{C}$ and $60 \%$ relative humidity in a rotating incubator. Newly hatched chicks were kept on a 12/12-h light/dark cycle (lights on at 8.00 a.m.) at $\sim 25^{\circ} \mathrm{C}$ and had access ad libitum to water and Superiniciarina chick starter (Gallina Blanca Purina, Madrid, Spain) as the carotenoid normal diet. Twenty chicks were used at ages of between 1 day (P1) and 60 days (P60) post-hatching. They were anaesthetized by inhalation of diethyl ether before decapitation. The animal care and handling protocols used in our laboratory and university vivaria conform to appropriate national legislation (Decree 223/1988, BOE no. 67) and the guidelines of the Council of the European Communities (86/609/CEE).

\section{Photoreceptor isolation}

A cut through the equator of the eye globe was made with scissors. The whole central retina was removed and dissociated by enzymatic digestion following the procedure of López et al. (1997) with variations in order to obtain the largest number of entire photoreceptors (see Results). We used a protease preparation from Streptomyces fradiae (SF-protease) with a specific activity of approximately 24,000 modified Anson units per milligram, produced by us in the Center of Molecular Biology of Autonoma University of Madrid (Spain).

Fresh cell preparations

A $20-\mu 1$ drop of the cell suspension was placed on a slide, covered with a coverslip $(20 \times 20 \mathrm{~mm})$ and sealed with nail lacquer. Cells were observed by phase contrast with a Zeiss Universal photomicroscope equipped with a MC63A exposimeter and $\mathrm{M} 35 \mathrm{~W}$ camera. A blue filter was placed between the microscope lamp and the condenser to eliminate excess yellow incident light. Kodakcolor Gold 100 film was used because it reproduced the natural colours seen by direct observation better than other films (López et al. 2005). Panel illustrations were made by scanning the films (hp Scanjet 5470) and mounting of the selected images with Adobe Photoshop 7.0, with adjustments of contrast, brightness and colour for the faithful reproduction of the colours seen in the prints. Camera lucida drawings were made by using a Neofluar $\times 100$ oil lens. Photographs were taken via a Planapoachromatic $\times 40$ oil lens.

\section{Feulgen-stained photoreceptors}

Drops of the cell suspension were spread onto gelatincoated slides, air-dried, fixed in a mixture of ethanol-acetic acid (3:1) for $1 \mathrm{~h}$, washed for $15 \mathrm{~min}$ in distilled water and air-dried once more. For Feulgen staining (Feulgen and Rossenbeck 1924), slides were exposed to $5 \mathrm{~N}$ hydrochloric acid in a glass jar for $30 \mathrm{~min}$ at $19 \pm 1^{\circ} \mathrm{C}$, washed three times with distilled water at room temperature, treated with Schiff's reagent for $2.5 \mathrm{~h}$ in a humid chamber, and finally washed three times with distilled water for $10 \mathrm{~min}$ and dried. The original Feulgen method had three washes with a sulphite solution after treatment with Schiff's reagent but this is now known to be unnecessary. At the time of the study, a droplet of distilled water was placed on the cellspread area and coverslipped. Cells were examined under phase-contrast microscopy with a $\times 100$ oil lens and camera lucida drawings of photoreceptor shapes and their nuclei were made for reconstruction of the ONL. The Feulgen method specifically stains the DNA and thus outlines the nucleus shape. For methodological convenience, this was used instead of DAPI staining, since the fixation for Feulgen staining firmly fixes the cells to the slide, avoiding the loss of cells during the washings after fixation. Moreover, their shapes are preserved better than by $4 \%$ paraformaldehyde (PFA) used prior to DAPI staining, and staining and cell preservation are permanent without any mounting medium. Photographs were taken via a Planapoachromatic $\times 40$ oil lens.

\section{DAPI-stained sections}

The eyes were dissected and immersed in 4\% PFA in $0.1 \mathrm{M}$ phosphate buffer at $\mathrm{pH}$ 7.6. The corneas were immediately removed. After $1 \mathrm{~h}$ of fixation at room temperature, the fixative was renewed and fixation continued for at least $24 \mathrm{~h}$ at $4^{\circ} \mathrm{C}$. This was followed by three washes of $30 \mathrm{~min}$ in phosphate-buffered saline, cryoprotection by immersion of the eyes in $11 \%$ and $30 \%$ sucrose solution, successively, and sectioning at 7-8 $\mu \mathrm{m}$. Sections were mounted with a medium containing DAPI and o-phenylenediamine (both from Sigma-Aldrich) for nuclei staining and anti-fading, 
respectively. Sections were studied by fluorescence with a Zeiss photomicroscope and images were transferred to Adobe Photoshop 7.0 for plate montage.

\section{Cell counting}

The percentage of the Ramón y Cajal's cone types with distinct droplet colour and nucleus position was determined by using freshly dissociated cell preparations from P30 chick retinas. A total of 1,905 photoreceptors were counted in fields chosen at random. All entire photoreceptors in each field were counted and the droplet colour and the position of the soma with respect to the OLM of each one was annotated. Percentages of each type and sub-type were referred to either the total of photoreceptors or the total of cones. A Planapoachromatic $\times 40$ oil lens was used.

\section{Results}

\section{Dissociation of entire photoreceptors}

In the procedure by López et al. (1997) to dissociate entire cells from the central nervous system (CNS) of vertebrates with SF-protease, the protease concentration and time of incubation in the protease solution were given for the three regions of the CNS (retina, cerebellum, and hippocampus) and for the two animal species (chicken and rat) investigated. For the adult chicken retina, $76-152 \mu \mathrm{g} / \mathrm{ml} \mathrm{SF-}$ protease and 45-60 min of incubation were reported to be adequate to obtain a suspension of cells of all types (except ganglion cells), with their shapes being well preserved. However, the cell suspension achieved under these conditions contained many more Müller cells and amacrine and horizontal cells that were better preserved than photoreceptors and bipolar cells. Many of these last-mentioned cells were damaged, lacking the oil droplet and outer segment in the case of photoreceptors and the axon in the case of bipolar cells. In the present work, we investigated conditions to improve the number of entire photoreceptors obtained. Four factors were found to vary the results: the protease concentration, the time and temperature of the incubation in the protease solution and the intensity of the mechanical disruption used. The optimum conditions found were: $65-70 \mu \mathrm{g} / \mathrm{ml}$ SF-protease in $175 \mathrm{mM}$ sucrose solution in distilled water, $40 \mathrm{~min}$ of incubation at most, at $33-34^{\circ} \mathrm{C}$, and with mechanical dissociation at 10 and $20 \mathrm{~min}$ from the beginning of the incubation period, consisting of five aspirations of the cell suspension, smoothly made through the tip of a 1-ml Gilson pipette. After $40 \mathrm{~min}$ of incubation, digestion was stopped by cooling the cell suspension to $20-23^{\circ} \mathrm{C}$. This was left for $10 \mathrm{~min}$, after which gentle mechanical disruption was provided. Under these conditions, abundant entire photoreceptors were obtained with their natural orientation in the tissue generally being preserved (Figs. 1, 2). Variation of any of these factors clearly decreased the cell number, mainly when the mechanical disruption was not gentle. The outer segment of photoreceptors was difficult to preserve because of their connection to the inner segment by a cilium. The thin processes of the synaptic pedicle of cones were also difficult to preserve.

\section{Study of dissociated photoreceptor shapes}

The study of fresh and fixed dissociated photoreceptors showed mainly cones (Figs. 1a-k, 2b-g) and a few rods (Figs. 11, 2a). The four cone types described by Ramón y Cajal (1892) were identified and all but the accessory cone had larger somata than did the rods. The observation of small groups of photoreceptors joined only at the level of the OLM (not shown) revealed that the small protuberance observed at a short distance from the soma on the side of the inner segment in isolated cones (arrowheads in Figs. 1, $2,5)$ corresponded to the OLM, that is, this protuberance marked the inner limit of the inner segment. In straight cones, the soma was either close to the OLM, close to the synaptic body or at an intermediate position (Figs. 1a-g, $2 \mathrm{e}, \mathrm{f})$. Straight cones with the soma close to the synaptic body or at an intermediate position exhibited a short process between the OLM and the soma, hereafter called the inner process (Fig. 1b,c,e,g). Most of straight cones had an axon of variable length (Fig. 1a,b,d-g) but a few lacked an axon, the synaptic processes leaving directly from the soma (Fig. 1c); this finding contradicts the idea that all straight cones have an axon (Ramón y Cajal 1892). The two members of the double cones appeared frequently joined in couples (Fig. 2b), mainly in the experiments in which the final mechanical dissociation was gentle. This agrees with the notion that SF-protease digests the extracellular matrix proteins but not the cell-membrane proteins; thus, it does not digest the transmembrane proteins that form the gap junctions by which the cone couple is tightly joined. Clearly, the complete isolation of the principal and accessory cones was achieved by mechanical disruption. Surprisingly, the principal member was found to lack an axon in all the couples studied (at least 100). It had the largest soma (generally bottle-shaped) and synaptic processes leaving directly from it (Figs. 1k, 2b,c). The accessory member had the smallest soma among cones and a thin axon with the pedicle generally at the level of the synaptic processes of the principal cone (Figs. 1j, 2b,d). A few cones had long axons with short lateral processes and the soma close to the OLM (Figs. 1i, 2g); these were undoubtedly the oblique cones described by Ramón y Cajal (1892). Therefore, we show some straight cones without an 

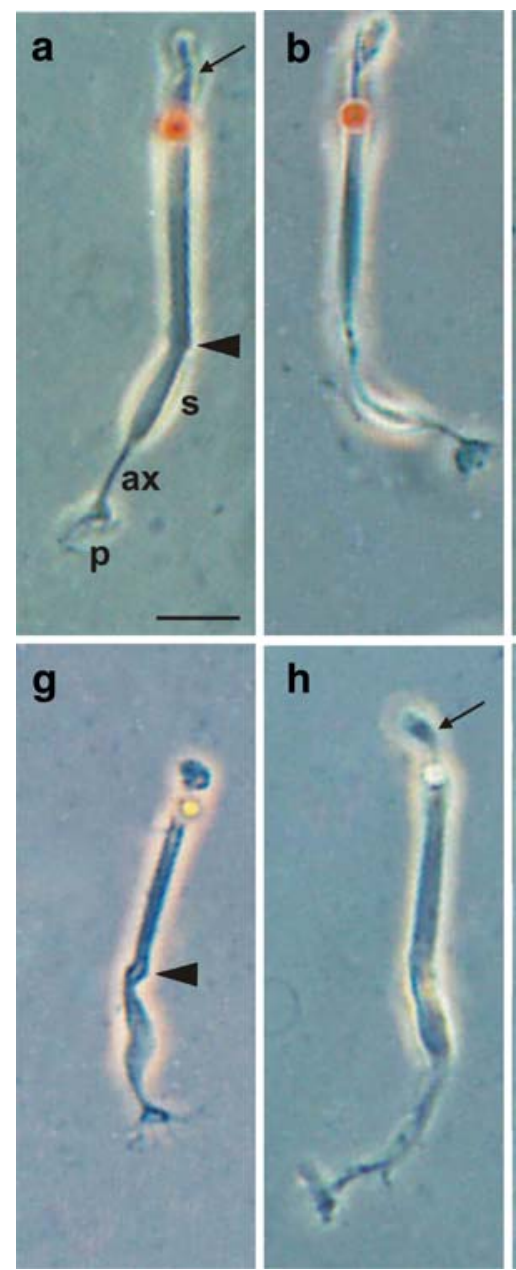

Fig. 1 Phase-contrast photomicrographs showing freshly isolated adult photoreceptors from P15-P60 chick retinas ( $s$ soma, ax axon, $p$ synaptic pedicle, thin arrow outer segment). Cones had a coloured oil droplet $(\mathbf{a}-\mathbf{k})$, whereas rods did not (l). Straight cones $(\mathbf{a}-\mathbf{h})$ differed by the droplet colour (red, orange, yellow, colourless) and position of the soma $(s)$ with respect to the outer limiting membrane (OLM; arrowheads). The soma of straight cones with a red droplet lay in outer (a), middle (b) and inner (c) positions; the soma of those with an orange droplet, in outer (d) and middle (e) positions; the soma of

axon and all principal cones also without an axon. All cones had an oil droplet, that of the accessory cone being small (Fig. 1j) and thus not visible until P3-P4 with a $\times 40$ objective. Rods had a small soma (oval or rounded), lacked an axon and oil droplet and had generally thinner inner segments and longer outer segments than did cones.

The study of the droplet colour of each cone type in freshly dissociated photoreceptor preparations showed that, whereas straight cones had the whole variety of droplet colours (red, orange, different tonalities of yellow and colourless), the principal cone only had colourless and yellow droplets, the oblique cone only colourless droplets and the accessory cone pale green droplets (Fig. 1), this last colour not being properly reproduced in the micrographs. Furthermore, we observed that the percentage of the

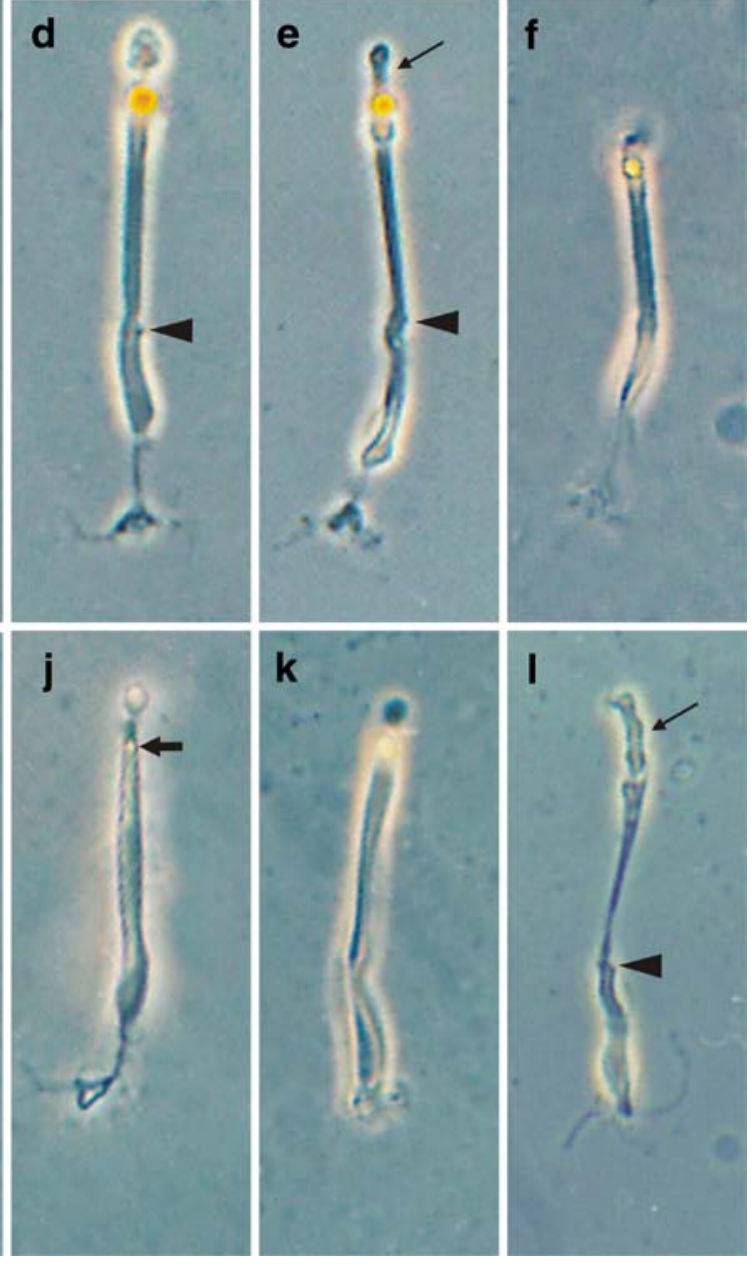

those with a yellow droplet, also in outer (f) and middle (g) positions; the soma of those with a colourless droplet, only in the outer position (h). Oblique cones only showed a colourless droplet and the soma in the outer position (i). Accessory cones had a small green droplet (thick arrow) and the soma in the outer position (j). Principal cones had a light-yellow (k) or colourless (not shown) droplet and the soma extended from the OLM to the pedicle. Note that some straight cones with a red droplet (c) and the principal cones (k) lacked an axon, their pedicle stemming directly from the soma. Bar $10 \mu \mathrm{m}$

different droplet colours varied in straight and principal cones. The large majority of straight cones had red and orange droplets, whereas the large majority of principal cones had yellow droplets. Therefore, we determined the percentage of cones with different droplet colour (Table 1). We referred their percentages to the total number of cones and included those with pale-yellow, lemon-yellow and bright-yellow droplets in the single group of yellow. As we did not see the large green droplets detected in a previous study of fresh whole-mount retinal preparations (López et al. 2005), we concluded that the tissue changes the colour appearance of some droplets. We studied a total of 1,905 dissociated photoreceptors, finding $82 \%$ with an oil droplet (i.e. cones), against $18 \%$ without a droplet and with characteristics of rods. Straight cones made up $36.5 \%$ of 

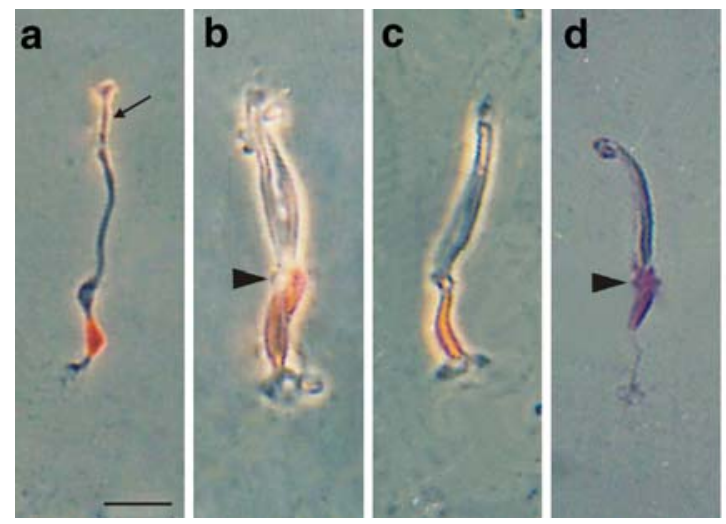

Fig. 2 Photomicrographs showing isolated adult photoreceptors from P1-P15 chick retinas with their nucleus stained by the Feulgen method (red or purple). a-c, e-g Phase contrast. d Transmitted light with the condenser diaphragm nearly closed to discern the cell contour. a Rod. b Double cone (arrowhead position of OLM) with the principal member (left) and the accessory member (right). c Principal

photoreceptors, double cones (principal and accessory types) $44 \%$ of photoreceptors, and oblique cones only $1.5 \%$ of photoreceptors. Straight cones with red and orange droplets comprised $19.5 \%$ and $17 \%$ of cones, respectively, those with yellow droplets representing fewer $(7.69 \%)$ and those with colourless droplets being rare $(0.13 \%)$. All oblique cones were found to have colourless droplets and only accounted for $1.66 \%$. Principal cones had mostly yellow droplets $(25.56 \%)$, and only a few had a colourless droplet (1.41\%). Accessory cones accounted for $26.97 \%$ and each had a small greenish droplet.

The study of the position of the soma of cones with different droplet colour and their percentages (Table 1) showed that straight cones with a red droplet had the soma in outer, middle and inner positions, those with an orange or yellow droplet had the soma in outer and middle
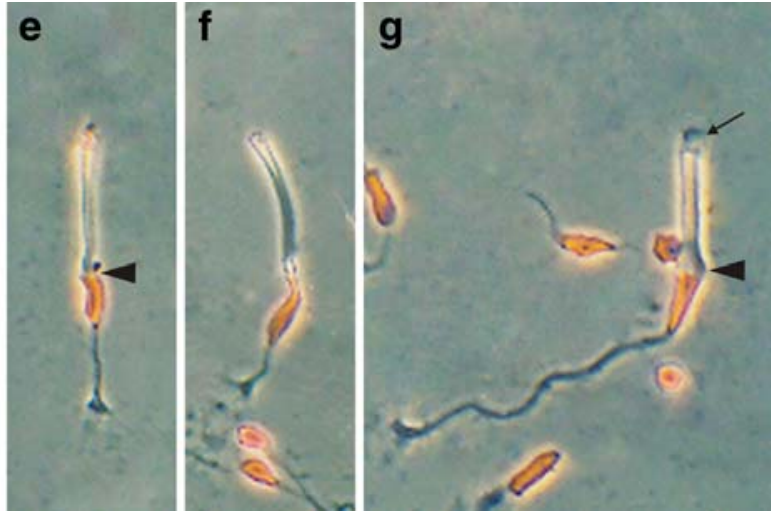

cone similar to that in Fig. 1k. Note that its nucleus extends from the OLM to the synaptic pedicle. d Accessory cone showing a portion of its nucleus outside the OLM (arrowhead). e, f Straight cones with the nucleus in the outer and middle positions, respectively (arrowhead position of OLM). g Oblique cone (arrowhead position of OLM). Arrow points to outer segment. Bar $10 \mu \mathrm{m}$

positions and those with a colourless droplet had the soma only in the outer position. The majority of straight cones had the soma in the outer position, with those with a red or orange droplet dominating $(12.75 \%$ and $12.62 \%$, respectively), followed by those with a yellow droplet (6.47\%), and then those with a colourless droplet $(0.13 \%)$. Among those with the soma in middle position, the majority also showed a red or orange droplet $(6.27 \%$ and $4.42 \%$, respectively), and a few had a yellow droplet (1.22\%). A few straight cones had the soma in the inner position and exhibited only a red droplet $(0.51 \%)$. All oblique cones had the soma in the outer position (1.66\%). All principal cones had the soma in the trans-layer position $(26.97 \%)$, since it extended from the OLM to the synaptic processes. All accessory cones had the soma in the outer position (26.97\%). Therefore, the results in Table 1 and Figs. 1, 2
Table 1 Percentages of chick photoreceptors in freshly dissociated cell preparations from $\mathrm{P} 15-\mathrm{P} 60$ retinas. The percentages of class (column 2) and type (column 4) of photoreceptors refer to the total number of photoreceptors $(n=1,905)$, whereas the percentages of a specific droplet colour (column 6) and soma position (column 8) refer to the total number of cones $(n=1,561)$

\begin{tabular}{|c|c|c|c|c|c|c|c|c|}
\hline Class & Class \% & Type & Type \% & Droplet colour & Droplet colour \% & Soma position & Soma position $\%$ & Cell number \\
\hline \multirow[t]{12}{*}{ Cones } & \multirow[t]{12}{*}{82} & \multirow[t]{8}{*}{ Straight } & \multirow[t]{8}{*}{36.5} & \multirow[t]{3}{*}{ Red } & \multirow[t]{3}{*}{19.54} & Outer & 12.75 & 199 \\
\hline & & & & & & Middle & 6.27 & 98 \\
\hline & & & & & & Inner & 0.51 & 8 \\
\hline & & & & \multirow[t]{2}{*}{ Orange } & \multirow[t]{2}{*}{17.04} & Outer & 12.62 & 197 \\
\hline & & & & & & Middle & 4.42 & 69 \\
\hline & & & & \multirow[t]{2}{*}{ Yellow } & \multirow[t]{2}{*}{7.69} & Outer & 6.47 & 101 \\
\hline & & & & & & Middle & 1.22 & 19 \\
\hline & & & & Colorless & 0.13 & Outer & 0.13 & 2 \\
\hline & & Oblique & 1.5 & Colorless & 1.66 & Outer & 1.66 & 26 \\
\hline & & \multirow[t]{2}{*}{ Principal } & \multirow[t]{2}{*}{22} & Yellow & 25.56 & Trans-layer & 25.56 & 399 \\
\hline & & & & Colorless & 1.41 & Trans-layer & 1.41 & 22 \\
\hline & & \multirow[t]{2}{*}{ Accessory } & \multirow[t]{2}{*}{22} & Green & \multirow[t]{2}{*}{26.97} & Outer & \multirow[t]{2}{*}{26.97} & 421 \\
\hline Rods & 18 & & & No droplet & & Inner & & 344 \\
\hline
\end{tabular}


indicate that the outer row of the ONL contains the soma of the majority of straight cones and all accessory cones, at a proportion of approximately $1: 1$, the few somata of the oblique cones and a small portion of the soma of all principal cones. The middle row of the ONL possesses the somata of about a quarter of the straight cones and a portion of the soma of all principal cones. The inner row has the soma of all rods, the largest part of the soma of all principal cones, and the soma of a few straight cones. These results suggest that the middle and inner rows of the ONL have a lower number of nuclei than the outer row. The study of DAPI-stained sections supports this view, as shown below.

Study of the shape and position of nuclei in Feulgen-stained dissociated photoreceptors

In Feulgen-stained dissociated photoreceptor preparations, the shape of the nucleus and its position in the cell were clearly observed (Fig. 2). We studied hundreds of photoreceptors and drew many of them with the different nucleus shapes in each of the three positions, aligning them with respect to the OLM. A representative sample is shown in Fig. 3a.

Rods had oval or rounded nuclei (Figs. 2a, 3a1), whereas cone nuclei had various shapes including oval, spindle, bottle, elongated and triangular (Figs. 2b-g, 3a2-8).
Principal cones generally had bottle-shaped nuclei extending from the OLM to the synaptic processes, with a thin and curved neck in the outermost part and an enlarged body in the middle and inner portions (Figs. 2b,c, 3a2). Accessory cones had small nuclei (compared with those of the principal cones) in an outer position, in general with an indentation at the level of the OLM and a small part of it protruding inside the inner segment (Figs. 2b,d, 3a3). Straight cones had nuclei mainly of spindle and elongated shapes in outer (Figs. 2e, 3a4, 5), middle (Figs. 2f, 3a6, 7) and inner positions (rare, not shown). Oblique cones had triangular nuclei in outer positions (Figs. 2g, 3a8).

Taking into account the type of cones found in the three rows of the ONL and their percentages (Table 1) and considering that rods lay in the inner row, we reconstructed the ONL with the drawn nuclei (Fig. 3b). The constructed ONL showed a continuous outer row and discontinuous intermediate and inner rows, agreeing with the image of the ONL of the central and equatorial retina taken from DAPI-stained sections (Fig. 4a,b,e,f).

\section{Study of the ONL in DAPI-mounted sections}

Transverse sections from the nasal and temporal retina of P25 chicks were used and whole sections studied. The ONL had three rows of nuclei (outer, middle and inner rows) in the central and equatorial retina (Fig. 4a,b,e,f), whereas it

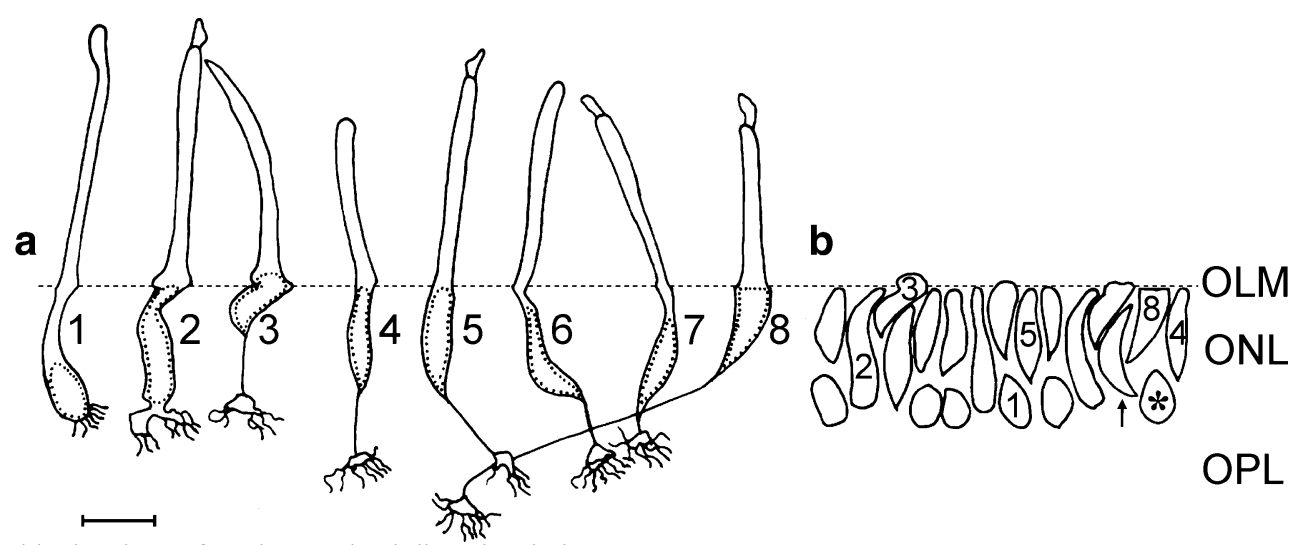

Fig. 3 Camera lucida drawings of Feulgen-stained dissociated photoreceptors, from P15 retinas. a Selection of photoreceptors representative of rods (1) and cones (2-8) aligned with respect to the OLM (OLM, horizontal dotted line) and showing the shapes and positions of their nuclei (dotted shapes). Rods had small nuclei (oval or rounded) close to the synaptic processes and long inner processes (the portion of the cell from the outer pole of the soma to the OLM). Principal cones (2) showed bottle-shaped nuclei extending from the OLM to the pedicle. Accessory cones (3) had a nucleus with a typical indentation and a portion of it inside the inner segment (the portion of cell from the OLM to the outer segment). In straight cones, the nuclei lay either close to the $\operatorname{OLM}(4,5)$ or at some distance from it $(6,7)$; these cones had a short inner process compared with that of rods. Note that these cones had the nucleus in the middle position with respect to that of the nucleus of rods and accessory cones. Straight cones with the nucleus close to the synaptic processes and thus without an axon (as in Fig. 1c) were not drawn because they were not clearly distinguished from rods because the oil droplet was not visible in Feulgen-stained cells. Oblique cones ( 8 ) showed a typical triangular-shaped nucleus and the longest axon among cones. b Reconstruction of the ONL by transportation of nuclei of drawn cells to the corresponding position. The nuclei of photoreceptors $1-5$ and 8 in a were used for this reconstruction, being indicated by the corresponding numbers (arrow a nucleus in middle position and representing cones 6 and 7 in a). The asterisk indicates a nucleus representing a straight cone without an axon and with a red oil droplet (Fig. 1c); this would be aligned with rod nuclei in the innermost position of the ONL, since both had their nuclei close to the synaptic processes $(O N L$ outer nuclear layer, $O P L$ outer plexiform layer). Bar $6 \mu \mathrm{m}$ 

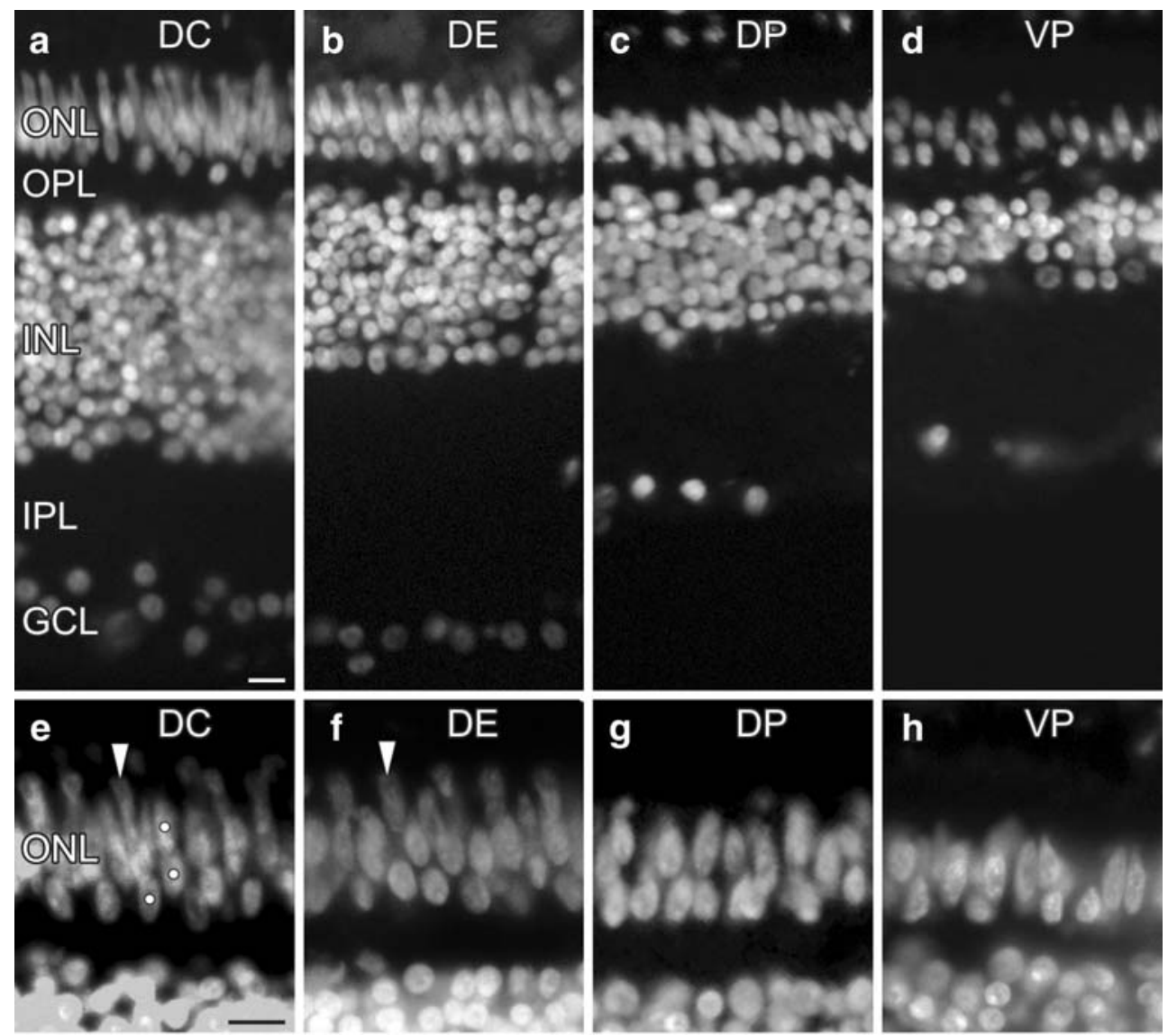

Fig. 4 Transverse sections from P25 retina fixed in 4\% PFA and mounted with DAPI, showing the nuclei of the retinal layers. a-d Fields from the same section, at low magnification, from the central to peripheral retina. Note that the number of nuclei in the ganglion cell layer $(G C L)$ and retinal thickness decreases. Three rows of nuclei can be seen in the outer nuclear layer $(O N L)$ of the central and equatorial retina and two rows in the ONL of the peripheral retina $(O P L$ outer plexiform layer, $I N L$ inner nuclear layer, $I P L$ inner plexiform layer). a

had two rows of nuclei in the peripheral retina (Fig. 4c,d,g,h) or even one row in the most peripheral retina (Fig. 4d,h, right). Furthermore, the nuclei in the peripheral retina were clearly less crowded than in the central and equatorial retinas (Fig. 4c,d,g,h), in agreement with the thick outer processes of Müller cells in the peripheral retina (Anezary et al. 2001). The ONL of the central retina showed a continuous outer row of nuclei with oval, elongated, or triangular shapes, a middle row of elongated or oval nuclei, and an inner row of oval or rounded nuclei that were regularly spaced (Fig. 4a,e), similar to the results that we had found by reconstructing the ONL from Feulgen-stained nuclei. Of note, the portion of the nucleus of the accessory cones that protruded into the inner segment was regularly spaced in the central and equatorial retina (Fig. 4, arrowheads), but this was not observed in the peripheral retina. No difference was found between nasal and temporal sections.
Dorsal-central $(D C)$ field close to the optic nerve head. b Dorsalequatorial $(D E)$ field. c Dorsal-peripheral $(D P)$ field. d Ventralperipheral $(V P)$ field close to the ora serrata. $\mathbf{e}-\mathbf{h}$ Fields equivalent to those in $\mathbf{a}-\mathbf{d}$ from sections at higher magnification showing mainly the ONL (dots in e nuclei in the inner, middle and outer rows, similar to the view in $\mathbf{f}$ ). $\mathbf{g}, \mathbf{h}$ Two rows of nuclei are visible, except in the region corresponding to the most peripheral retina in which nuclei form one row $(\mathbf{h}$, right $) . \quad B a r 10 \mu \mathrm{m}$
Cone diversity and their contribution to the ONL and OPL

The variety of chick cones is represented, in Fig. 5, on the basis of the oil droplet colour and soma position, which together with Table 1 provide an accurate idea of the quantitative contribution of each cone type to the ONL and OPL. From the combined study of both the representation in Fig. 5 and the data in Table 1, the following conclusions have been drawn. Two subtypes of principal cones exist according to their oil droplet colour, one with a yellow droplet and the other with a colourless droplet, the great majority having a yellow droplet. Accessory cones constitute a uniform population in terms of droplet colour, since all have a small greenish droplet. The nucleus of both principal and accessory cones has a restricted position, that of the principal cone has a trans-layer position, whereas that of the accessory cone has an outer position. Eight subtypes of straight cones occur as based on droplet colour 


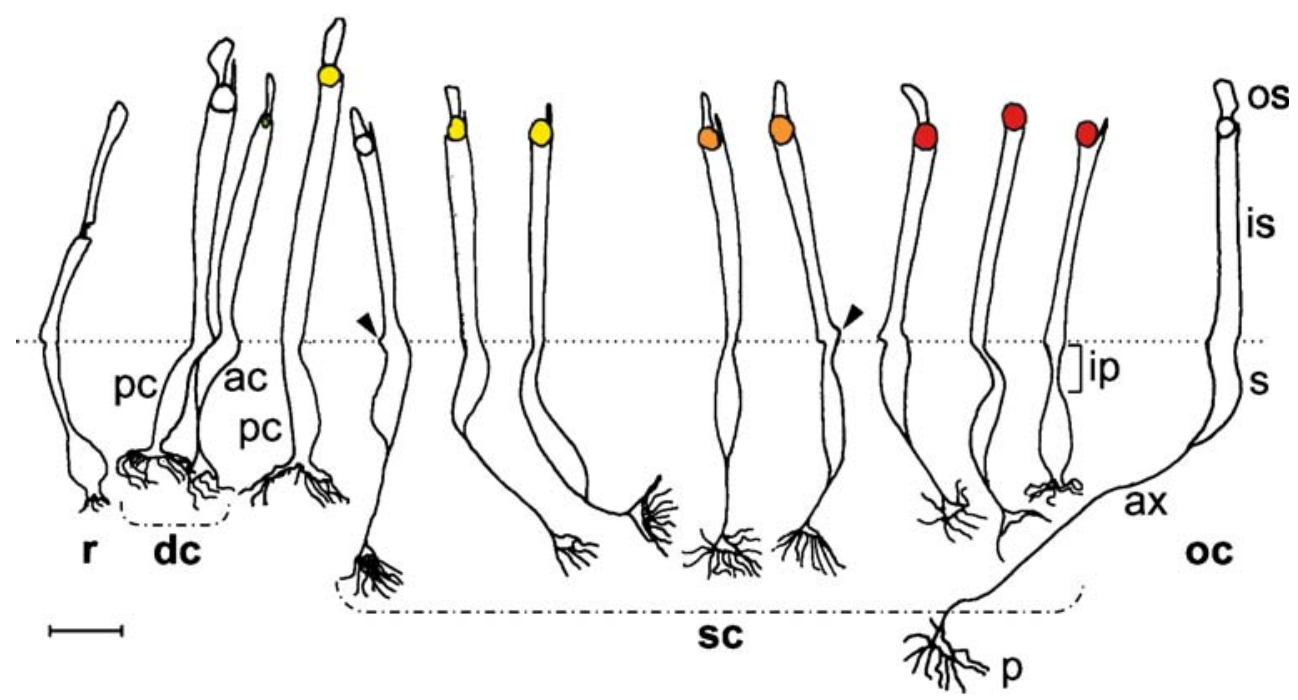

Fig. 5 Representation from camera lucida drawings of freshly dissociated cells, aligned with respect to the OLM (dotted line) and showing the photoreceptor types in $\mathrm{P} 15-30$ chick retina: rod $(r)$; double cone $(d c)$ consists of a principal member $(p c)$ having a colourless droplet and the accessory member (ac) having a green droplet; the great majority of principal cones however have a yellow droplet and are represented by the cone to the right of the double cone; straight cones $(s c)$ have a colourless, yellow, orange or red droplet and can be subdivided into two subtypes with a yellow or orange droplet and three subtypes with a red droplet depending on the position of their nucleus (ip inner process). Note the small protuberance (arrowheads) shown by most photoreceptors at the level of the OLM. The oblique cone (oc) has a colourless droplet (os outer segment, is inner segment, $s$ soma, $a x$ axon, $p$ synaptic pedicle). Bar $6 \mu \mathrm{m}$ and nucleus position. Those with a colourless droplet have the nucleus only in the outer position, those with a yellow droplet have the nucleus in the outer and middle positions, those with an orange droplet also have the nucleus in the outer and middle positions, and those with a red droplet have the nucleus in the outer, middle and inner positions. Oblique cones constitute a uniform population, all with a colourless droplet and with the nucleus in the outer position. Therefore, we have distinguished 12 types of cones.

The variation of the position of the nucleus in the straight cones seems to be related to the variation in the axon length and the presence or absence of the inner process (Fig. 5). Thus, cones with the nucleus in an outer position show the longest axon and lack inner processes, those with the nucleus in the middle position show a shorter axon and have a short inner process and those with the nucleus in the inner position do not show an axon and have the longest inner process. Since straight cones receive the name because of the straight disposition of their axon (Ramón y Cajal 1892), it is obvious that the axon length relates to the synaptic level of the pedicle in the OPL and, thus, those with the largest axons synapse in the innermost stratum of the OPL, whereas those without axons synapse in the outermost stratum. Consequently, the scheme suggests that the synaptic processes of the principal and accessory cones and those of the straight cones lacking an axon (with a red droplet) are in the outer stratum of the OPL, those of the straight cones with an axon are in the middle stratum and those with the oblique cones are in the inner stratum. These latter results agree with the scheme proposed by Gallego (1976) from an electron-microscopy study of the retina of diurnal birds.

\section{Discussion}

We show that cone diversity in the chicken retina is greater than previously thought as based on the oil droplet colour and nucleus position of the cone morphological types described by Ramón y Cajal (1892). This diversity suggests functional and identity differences. Chick cones are distinguished functionally not only by the type of pigment in the outer segment and the type of oil droplet, but also by their synaptic connectivity, like other retinal cells. Since the morphological type of cones correlates with the OPL stratum in which they form synapses, i.e. double cones in the outer stratum, straight cones in the intermediate stratum and oblique cones in the inner stratum, and since different bipolar cells synapse with them in each stratum (Gallego et al. 1975; Ramón y Cajal 1892), knowledge of the visual pigment and the colour and microspectrophotometric characteristics of the droplet of each morphological cone type appears to be necessary in order to provide a more complete functional scheme of cones. According to the droplet colour, we have distinguished eight cone types, of which four are straight cones (Table 1). The droplet colours detected by us coincide with those described by Goldsmith et al. (1984) who have distinguished three yellow droplet types, "yellow, pale yellow or greenish, and pale yellow or 
colourless", which we have grouped in a single yellow pool, plus a transparent droplet without significant absorbance that might correspond to our colourless droplet. However, Goldsmith et al. (1984) have not correlated droplet colours with morphological cone types.

Preceding studies that have assigned droplet colour to morphological cone types in the chicken retina (all using retinal whole-mounts) show considerable disagreement with regard to their results. We express these results together with our data in Table 2 in order to facilitate this discussion. Preceding researchers have assigned a yellow droplet to principal cones, with the exception of Oishi et al. (1990), who have assigned them a pale green droplet. Our results show that the majority of principal cones have a yellow droplet but that a few have a colourless droplet, suggesting two functional types of principal cones. However, microspectrophotometric studies have revealed red pigment and pale droplets (P-type) in principal cones (Bowmaker et al. 1997), indicating a single functional type. Principal cones with colourless droplets may have escaped the notice of previous researchers because of their low percentage. The microspectrophotometric study of the visual pigment and oil droplet in the isolated principal cones with a colourless droplet would determine whether they are a functional type differing from principal cones with a yellow droplet. Some researchers have found the droplet of the accessory cone to be greenish or green (Gallego 1976; Meyer and Cooper 1966; Meyer and May 1973), whereas others have found it to be yellow (Gayoso et al. 1978) or colourless (Gallego et al. 1975), and still others found no droplet or found minute droplets (Bowmaker et al. 1997; Oishi et al. 1990). In this study, we have detected a pale green droplet in all accessory cones of chicks from P7 onwards. Factors responsible for differences in droplet-colour detection have been discussed elsewhere (López et al. 2005) and therefore we will not repeat this information here. The failure by some researchers to find droplets in accessory cones can be fully explained by the age of the animals. We started to observe a small droplet at P3, with increasing numbers of accessory cones having a droplet from P4 to P7. In microspectrophotometric studies, Bowmaker et al. (1997) have reported a red-sensitive pigment in the outer segment of chick accessory cones but have not studied the droplet because of its small size and its apparent absence in some cones. In any case, accessory cones appear to be functionally of a single type, since they have a red-sensitive pigment, a droplet of a single colour and form synapses in the outer OPL stratum (Gallego 1976; Gallego et al. 1975; Ramón y Cajal 1892).

Most studies on the oil droplet colour of chick cones do not distinguish straight from oblique cones (Table 2). Since the great majority of single cones are straight (Table 1), the oil droplet colours reported for single cones correspond mainly to the straight cones. The disagreement is considerable: early studies assign only a red colour to single cones, whereas later studies assign them red, yellow-green, orange, yellow, bright blue and clear. We show straight cones with red, orange, yellow and colourless droplets, which might correspond to the four microspectrophotometric droplet types found in single cones on the basis of the $\lambda_{\text {T50 }}$ (Bowmaker and Knowles 1977) or the $\lambda_{\text {cut }}$ (Bowmaker et al. 1997). Since each cone with a spectrophotometric droplet type has a pigment type (Bowmaker et al. 1997), four functionally different single cone types exist: red, green, blue and violet, according to their pigment. Straight cones are clearly of these four functional types but this must be demonstrated by determining the pigment type in each isolated straight cone of a different droplet colour. The different percentages of straight cones with distinct droplet colours (Table 1) indicate that they contribute differently to the function of the middle stratum of the OPL.

Two studies with fresh chick retinal whole-mounts have assigned a green or yellow oil droplet to oblique cones (Gallego 1976; Gayoso et al. 1978). Although micro-

Table 2 Oil droplet colour in chick cones from previous studies and our present results ( $N A$ not applicable)

\begin{tabular}{|c|c|c|c|c|c|}
\hline Reference & Principal & Accessory & Single & Straight & Oblique \\
\hline Meyer and Cooper 1966 & Golden-yellow & Greenish & Red & NA & NA \\
\hline Meyer and May 1973 & Golden-yellow & Yellowish-green & Red & NA & NA \\
\hline Gallego et al. 1975 & Yellow & Colourless & Red, yellow-green & NA & NA \\
\hline Gallego 1976 & Yellow-orange & Greenish & NA & Red & Green \\
\hline $\begin{array}{l}\text { Bowmaker and } \\
\text { Knowles } 1977\end{array}$ & Yellow-green & Yellow-green & $\begin{array}{l}\text { Red, orange-yellow, } \\
\text { bright blue, yellow-green }\end{array}$ & NA & NA \\
\hline Gayoso et al. 1978 & Yellow & Yellow & NA & Red, green & Yellow \\
\hline Szel et al. 1985 & Yellow-green & No colour indicated & Red, yellow & NA & NA \\
\hline Oishi et al. 1990 & Pale green & No droplet found & Red, yellow, pale-blue, Clear & NA & NA \\
\hline Our present results & Yellow and colourless & Green & NA & $\begin{array}{l}\text { Red, orange, } \\
\text { yellow, colourless }\end{array}$ & Colourless \\
\hline
\end{tabular}


spectrophotometric studies have not considered the oblique cone (Bowmaker et al. 1997; Bowmaker and Knowles 1977; Goldsmith et al. 1984), this cone type has evidently been studied within the group of single cones. We show that the oblique cone has a colourless droplet, which would correspond to either $\mathrm{C}$ or $\mathrm{T}$ microspectrophotometric droplet types, according to the results of Goldsmith et al. (1984) and Bowmaker et al. (1997) and it would have either blue-sensitive pigment or violet-sensitive pigment, on the basis of the correlation of droplet types and pigments made by the latter authors. To confirm this would require a microspectrophotometric study of the oil droplet and pigment of isolated oblique cones. The finding that these cones are the only cone type making synapses in the innermost stratum of the OPL with one of the three bipolar types (Gallego 1976; Gallego et al. 1975; Ramón y Cajal 1892) suggests that they are a functional type different from double or straight cones. Their low percentage would not necessarily minimize their functional importance, since other rare retinal cells known as displaced ganglion cells $(0.43 \%$ of the total number of ganglion cells), which have their soma between those of the amacrine cells and are more abundant in peripheral than in ventral retina (Prada et al. 1992), were initially thought to have no function and were later found to be involved in oculomotor reflexes and visuomotor movements. Of note, a small percentage of principal and straight cones also have a colourless droplet (Table 1) and thus might have blue or violet pigment. Therefore, our results together with those of other researchers suggest that three morphological cone types, viz. the oblique, principal and straight types bearing a colourless droplet and blue or violet pigment (the three types of low percentage), each form synapses in one of the three synaptic strata of the OPL.

We show that the principal cone lacks an axon, in agreement with the shapes drawn by others (Gallego 1976; Gayoso et al. 1978; Morris and Shorey 1967; Araki et al. 1984) but in contrast to the description of the principal cone by Ramón y Cajal (1892). In all the cone couples studied, only the accessory cones are seen to have an axon and, furthermore, the percentage of principal and accessory cones found $(26.97 \%$ each) is similar to that found by Oishi et al. (1990), viz. 26.6\%. We also show straight cones without an axon and with a red droplet; these might form synapses in the outer OPL stratum (Fig. 5). This agrees with the suspicion of Gallego et al. (1975) that some synaptic terminals of unidentified cones in this stratum are of single cones (referring to straight cones). Therefore, we show two cone types without an axon. The fact that Ramón y Cajal (1892) did not describe straight cones without an axon can be explained by their morphology being similar to that of rods and the impossibility of distinguishing them in Golgi preparations, since droplets are not observed in these preparations. A compelling question is why straight cones without an axon have only red droplets, whereas straight cones with an axon have any droplet colour and differ in percentage. Another intriguing question is why the percentages of cone types with different droplet colour varies.

Our results show that, in the central retina, three rows of nuclei exist in the ONL. All accessory cones and oblique cones and some straight cones with red, orange, yellow or colourless droplets have their nuclei in the outer row of the ONL. Some straight cones with red, orange, or yellow droplets are found in the middle row. Rods and some straight cones with red droplets occur in the inner row. All principal cones are present with a trans-layer disposition. Outer, middle and inner rows of nuclei are also clearly visible in the electron-microscopy study of Morris and Shorey (1967; plate 1 of their Fig. 4), although they have drawn two rows in their Fig. 1. The principal cone shows the largest nucleus, which contrasts with its small size in Fig. 1 of Morris and Shorey (1967) and Fig. 60 of Gallego (1976). These authors have used non-serial electronmicroscopy sections in which the entire cell nucleus is not visible. Our results also indicate that the percentage of nuclei of each cone type with distinct oil droplet colour in the three ONL rows differs notably. Although the studies in Drosophila mutants suggest a genetic control of the different nuclear positions of straight cones, the specification of the nuclear position by environmental cues, mostly spatial, is another possibility to consider.

Acknowledgments The authors thank Angela Amores for technical assistance in the preparation of histological specimens.

Open Access This article is distributed under the terms of the Creative Commons Attribution Noncommercial License which permits any noncommercial use, distribution, and reproduction in any medium, provided the original author(s) and source are credited.

\section{References}

Anezary L, Medina JI, Sanchez-Nogueiro J, López-Gallardo M, Prada C (2001) Shape diversity among chick retina Müller cells and their postnatal differentiation. J Comp Neurol 438:32-49

Araki M, Watanabe K, Yasuda K (1984) Immunocytochemical localization of rhodopsin-like immunoreactivity in the outer segments of the rods and single cones of chick retina. Cell Struct Funct 9:1-12

Bowmaker JK (1980) Colour vision in birds and the role of oil droplets. Trends Neurosci 3:196-199

Bowmaker JK, Knowles A (1977) The visual pigments and oil droplets of the chicken retina. Vision Res 17:755-764

Bowmaker JK, Heath LA, Wilkie SE, Hunt DM (1997) Visual pigments and oil droplets from six classes of photoreceptor in the retinas of birds. Vision Res 37:2183-2194

Dowling JE (1987) The retina. An approachable part of the brain. Belknap, Cambridge, Mass., USA 
Feulgen R, Rossenbeck H (1924) Mikroskopisch-chemischer Nachweis einer Nucleinsäure vom Typus der Thymonucleinsäure und die darauf beruhende selektive Färbung von Zellkernen in mikroskopischen Präparaten. Hoppe-Seylers Z Physiol Chem 135:203-248

Gallego A (1976) Comparative study of the horizontal cells in the vertebrate retina: mammals and birds. In: Zettler F, Weiler $\mathrm{R}$ (eds) Neural principles in vision. Springer, Berlin Heidelberg New York, pp 26-62

Gallego A, Baron M, Gayoso M (1975) Organization of the outer plexiform layer of the diurnal and nocturnal bird retinae. Vision Res 15:1027-1028

Gayoso MJ, Diaz-Flores L, Garrido M (1978) Gotas lipídicas de los fotorreceptores de la retina de los vertebrados. Morfol Normal Patol 2:1-28

Goldsmith TH, Collins JS, Licht S (1984) The cone oil droplets of avian retinas. Vision Res 24:1661-1671

Hart NH (2001) The visual ecology of avian photoreceptors. Prog Retin Eye Res 20:675-703

Inglehearn CF, Morrice DR, Lester DH, Robertson GW, Mohamed MD, Simmons I, Downey LM, Thaung C, Bridges LR, Paton IR, Smith J, Petersen-Jones S, Hocking PM, Burt DW (2003) Genetic, ophthalmic, morphometric and histopathological analysis of the retinopathy globe enlarged (rge) chicken. Mol Vis 9:295-300

López R, López-Gallardo M, Medina JI, Ramos M, Ramirez G, Prada C (1997) A Streptomyces fradiae protease dissociates structurally preserved neurons and glial cells from the embryonic and adult central nervous system of vertebrates. J Neurosci Methods 73:9-16

López R, López-Gallardo M, Busturia I, Anezary L, Prada C (2005) Spatial and temporal patterns of growth and differentiation of cone oil droplets in the chick retina. J Neurosci Res 79:401-411

Mariani AP, Leure-duPree AE (1978) Photoreceptors and oil droplet colors in the red area of the pigeon retina. J Comp Neurol $182: 821-837$

Meyer DB, Cooper TG (1966) The visual cells of the chicken as revealed by phase contrast microscopy. Am J Anat 118:723-734
Meyer DB, May HC Jr (1973) The topographical distribution of rods and cones in the adult chicken retina. Exp Eye Res 17:347355

Montiani-Ferreira F, Fischer A, Cernuda-Cernuda R, Kiupel M, DeGrip WJ, Sherry D, Cho SS, Shaw GC, Evans MG, Hocking PM, Petersen-Jones SM (2005) Detailed histopathologic characterization of the retinopathy, globe enlarged (rge) chick phenotype. Mol Vis 11:11-27

Morris VB (1970) Symmetry in a receptor mosaic demonstrated in the chick from the frequencies, spacing and arrangement of the types of retinal receptor. J Comp Neurol 140:359-398

Morris VB, Shorey CD (1967) An electron microscope study of types of receptor in the chick retina. J Comp Neurol 129:313-340

Oishi T, Kawata A, Hayashi T, Fukuda Y, Shichida Y, Yoshizawa T (1990) Immunohistochemical localization of iodopsin in the retina of the chicken and Japanese quail. Cell Tissue Res 261:397-401

Prada C, Medina JI, López R, Genis-Galvez JM, Prada FA (1992) Development of retinal displaced ganglion cells in the chick: neurogenesis and morphogenesis. J Neurosci 12:3781-3788

Ramón y Cajal S (1892) La rétine des vertébrés. La cellule 9:121-255

Smith RL, Nishimura Y, Raviola G (1985) Interreceptor junction in the double cone of the chicken retina. J Submicrosc Cytol 17:183-186

Szel A, Takacs L, Monostori E, Vigh-Teichmann I, Röhlich P (1985) Heterogeneity of chicken photoreceptors as defined by hybridoma supernatants. An immunocytochemical study. Cell Tissue Res 240:735-741

Vorobyev M (2003) Coloured oil droplets enhance colour discrimination. Proceedings 270:1255-1261

Whited JL, Cassell A, Brouillette M, Garrity PA (2004) Dynactin is required to maintain nuclear position within postmitotic Drosophila photoreceptor neurons. Development 131:4677-4686

Zhang T, Tran S, Clouser C, Pignoni F (2005) Nicastrin controls aspects of photoreceptor neuron specification and differentiation in the Drosophila eye. Dev Dyn 234:590-601 